\title{
Multi-diagnostic approach to Geodezic Acoustic Mode study
}

\section{A.Yu.Yashin*1, V.V. Bulanin ${ }^{1}$, A.V. Petrov' ${ }^{1}$, M.A. Petrov' ${ }^{1}$, V.K. Gusev², N.A. Khromov' ${ }^{2}$, G.S. Kurskiev ${ }^{2}$, M.I. Patrov ${ }^{2}$, Yu.V. Petrov ${ }^{2}$, S.Yu. Tolstyakov ${ }^{2}$, D.V. Prisyazhnyuk ${ }^{3}$}

${ }^{1}$ St. Petersburg Polytechnic University, St. Petersburg, Russian Federation

${ }^{2}$ Ioffe Institute, RAS, St. Petersburg, Russian Federation

${ }^{3}$ Max-Planck-Institut für Plasmaphysik, Garching, Germany

E-mail: alex_yashin@list.ru

\begin{abstract}
The phenomenon of zonal flows and geodesic acoustic modes (GAMs) in fusion devices is generally accepted to be a result of nonlinear interaction of small-scale drift waves. Therefore, the zonal flow and GAM study needs using diagnostic methods of plasma turbulence and plasma flow investigations. Moreover, these methods have to be employed for studying plasma density and magnetic field oscillations associated with the GAM excitation. In this report, a multi-diagnostic approach developed for the GAM research in the spherical tokamak Globus-M is described. Doppler backscattering (DB) method as the main tool for the GAM study, together with the diagnostics of plasma density and magnetic field GAM oscillations, were simultaneously used in experiments. The peculiar ray tracing analysis of the incident beam was previously performed to satisfy the DB specific requirements in a spherical tokamak which is characterized by a significant pitch angle at the low magnetic field side. The version of the DB diagnostics with two cut-offs positioned at different poloidal angles of the minor cross-section was employed in Globus-M. For the GAM plasma density oscillation study, the $D_{\alpha}$ emission was observed at different angles. Application of this diagnostic set-up together with the DB method allowed us to restore the spatial mode structure of the GAM velocity and plasma density oscillations. At the same time, the array of Mirnov coils was used for the GAM-like magnetic oscillation study, and that made it possible to determine the magnetic field perturbation spatial structure. The coherent and cross-bicoherence analyzes were employed to identify the interaction between the GAM oscillation of various plasma parameters and plasma turbulent fluctuations.
\end{abstract}

First EPs Conference on Plasma Diagnostics - 1st ECPD,

14-17 April 2015

Villa Mondragone, Frascati (Rome) Italy

*Speaker. 


\section{Introduction}

Geodesic acoustic modes (GAMs) as a class of highfrequency zonal flows manifest in tokamak plasma as localized flows in the direction of ExB drift in radial electric field [1]. The geodesic acoustic mode phenomenon is accompanied by plasma density oscillations at the GAM frequency. Moreover, the GAM theory predicts the GAM magnetic field oscillations [2]. Therefore, the GAM investigations involve application of various oscillation diagnostics of plasma parameters. Multi-diagnostic approach to the GAM study has been first employed on the T-10 tokamak [3] and then recently on the DIII-D [4] and TCV [5] tokamaks. This report describes a set of different GAM diagnostics in the spherical tokamak Globus-M and presents some results of the multi-diagnostic approach.

The main tool for the GAM flow study was Doppler backscattering (DBS) or the same Doppler reflectometry (DR) diagnostics [6]. The method allowed us to measure the ExB velocity oscillations and thus to reveal the GAMs. The peculiar

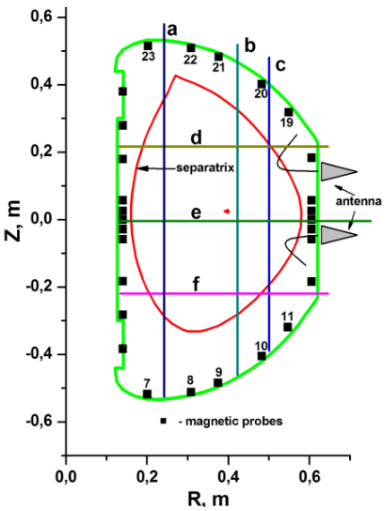

Fig. 1: Main diagnostics for GAM detection and ray tracing of DBS measurements in a minor cross-section of Globus-M. a-f are lines of sight for $D_{\alpha}$ emission detection; numbered squares are Mirnov coils ray tracing analysis of the incident beam was previously performed to satisfy the DBS specific requirements in a spherical tokamak characterized by a significant pitch angle at the low magnetic field side. The version of the DBS diagnostics with two cut-offs positioned at different poloidal angles of the minor cross-section was employed in Globus-M to study the spatial GAM velocity structure. The traditional diagnostics of plasma density such as Langmuir probes or reflectometry have limited opportunities in the research of the spatial structure of GAM plasma density oscillations. Due to this fact, we have proposed to use $D_{\alpha}$ emission detection. The observations of the $D_{\alpha}$ emission along six lines of sight were successively employed to restore the poloidal mode structure of plasma density. The spatial structure of magnetic field oscillations has been investigated in the tokamak Globus-M with the usage of Mirnov coils array. In conclusion, the methodological aspects of the DBS diagnostic data usage for bispectral analysis have been discussed. The conditions under which the bispectral analyses actually reveal the nonlinear mutual influence of GAM and drift turbulence have been identified.

\section{Set of GAM diagnostics on the Globus-M tokamak}

Multi-diagnostic approach has been applied for GAM investigation in the spherical tokamak Globus-M $(R=0.36 \mathrm{~m}, a=0.24 \mathrm{~m})$. The GAM has been discovered in Ohmic heating discharge $\left(<n>\approx(2-3) \cdot 10^{19} \mathrm{~m}^{-3}, I_{p}=150 \mathrm{kA}, B_{T}=0.4 \mathrm{~T}\right)$ when the direction of the magnetic field was selected in the way that the toroidal ion drift was directed away from the X-point [7]. The minor cross-section of the Globus-M and the separatrix are shown in Fig. 1

The main tool for GAM studies in the Globus-M tokamak was Doppler microwave backscattering. The method is based on the backscattering of microwave radiation at oblique incidence in the presence of a cutoff layer for the probing beam. Backscattering is mostly localized near 
the cutoff layer. The scattering occurs only when the Bragg condition is fulfilled that is when the scattering wave vector $k$ is perpendicular to the magnetic field lines in the vicinity of the reflection region. If so, it is possible to determine the component of the fluctuation velocity $V_{\perp}$ in the direction of the diamagnetic or ExB drift in the radial electric field by measuring the Doppler frequency shift $\Delta f_{D}=\mathbf{k} \cdot \mathbf{V}=k_{\perp} V_{\perp}$. According to the Bragg condition, $k_{\perp}=2 k_{0} \sin \alpha$, where $\alpha$ is angle of incidence of the probing microwave radiation onto the surface with constant refraction index at the plasma boundary and $k_{0}$ is the free space wavenumber. Therefore one can try to detect the GAM oscillation as an oscillation of the perpendicular velocity $V_{\perp}(t)$ or radial electric field (see Fig. $\left.2-E_{r}^{D R}\right)$. Moreover, the spectral power $\left|n\left(k_{\perp}, \omega\right)\right|^{2}$ of plasma density fluctuations for selected $k_{\perp}$-values can be estimated by measuring the backscattered power.

There are specific requirements for a DBS application in a spherical tokamak Globus-M which is characterized by significant pitch angle (over $20^{\circ}$ ) at the low magnetic field side. Therefore, to fulfill the Bragg condition, i.e. when the scattering wave vector is perpendicular to the magnetic field lines in a vicinity of the cut-off, the antenna had to be tilted in both poloidal and toroidal directions. To evaluate the needed antenna tilt angles, the incident beam propagation had to be determined. It was performed in quasi-optical approximation. The ray tracing of incident beam was obtained for the actual 3D geometry of Globus-M tokamak using the full dispersion expression for anisotropic plasma with allowance for spatial dispersion: $H\left(N_{\perp}^{2}, N_{\|}^{2}, v, u\right)=0, \quad v=\frac{\omega_{p e}^{2}}{\omega^{2}}, \quad v=\frac{\omega_{c e}^{2}}{\omega^{2}}$

Here $N_{\perp}^{2}$ and $N_{\|}^{2}$ are the perpendicular and parallel projections of normalized wave vector. $\omega_{p e}$ and $\omega_{c e}$ are the electron

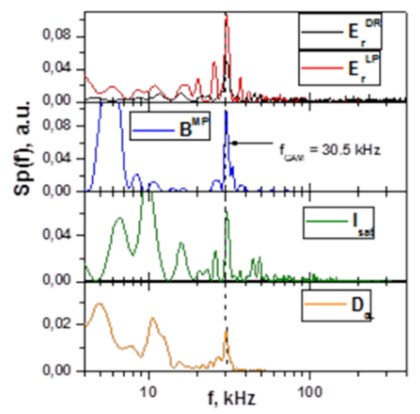

Fig. 2: Normalized auto-power spectra of radial electric field extracted by DR: $E_{r}^{D R}$ and Langmuir probes $E_{r}^{L P}$, GAM magnetic poloidal component $B^{M P}$, $D \alpha$ emission signal and probe ion saturation current $I_{\text {sat }}$ plasma and cyclotron frequencies respectively. This computation allows us to estimate the cut-off layer position and components of $k$ near the cut-off layer.

The version of the DBS layout with two microwave schemes with the cut-offs positioned at different poloidal angles $\left(-30^{\circ}\right.$ and $\left.45^{\circ}\right)$ of the same minor cross-section was employed in Globus$\mathrm{M}$ for the first time. Each reflectometer was based on a monostatic antenna scheme, which allowed probing the plasma by O-mode microwaves within the frequency band of 20 to $36 \mathrm{GHz}$. It was possible to change the incident frequency from shot to shot. The toroidal tilt angles for both reflectometers were in the range of $2^{\circ}-5^{\circ}$ and poloidal angles were of $6^{\circ}$ to $9^{\circ}$ respectively. The relevant wavelength of the scattering plasma fluctuations was in the range of $1.1-2.4 \mathrm{~cm}$. The cutoff positions ware in the vicinity of the separatrix at high $q$-values (in the interval of major radii $R$ from $53 \mathrm{~cm}$ to $58 \mathrm{~cm}$ ). The radial resolution of the method was estimated to be about $0.5 \mathrm{~cm}$. Dual homodyne detection [8] was applied to receive the backscattered radiation. The sine and cosine signals of the quadrature (IQ) detector were used for calculation of complex signal spectrum and subsequent determination of the Doppler frequency shift. The antennas and relevant ray tracing beams are schematically represented in Fig. 1. The two microwave schemes were designed to determine the phase relation between the GAM velocity oscillations at different poloidal angles. The location of the cut-offs on the same flux surface was controlled by the average rotation velocity 
measurements.

Plasma density and electric field oscillations at the GAM frequency were detected (see Fig. 2 $I_{s a t}$ and $E_{r}^{L P}$ ) by a row of four Langmuir probes placed in the mid-plane of the tokamak at the low magnetic field side. Each probe head was equipped with cylindrical tip $2.5 \mathrm{~mm}$ in diameter and 2 $\mathrm{mm}$ in length. The boron nitride isolators with high resistance were used in probes construction to ensure measurements near the separatrix. It was possible to move the probesalong the major radius in the range from $R=63 \mathrm{~cm}$ (wall) to $R=57 \mathrm{~cm}(1 \mathrm{~cm}$ inside the separatrix) from shot to shot. The probe row made it possible to detect simultaneously both the plasma density and the radial electric field oscillations by measuring the ion saturation current and the floating potentials of radially separated probes. Due to certain limitations of the Langmuir probes used for the GAM spatial structure study, they were mostly employed to determine the phase relationship between the plasma density and $D_{\alpha}$ emission oscillations.

The $D_{\alpha}$ emission oscillations at the GAM frequency were found on the tokamak Globus-M (see Fig. $2-D_{\alpha}$ ). These oscillations were treated as oscillations caused by the GAM density oscillations. Indeed, $D_{\alpha}$ emission could be dependent on plasma density, electron temperature and neutral density. However, there is no reason for the density of neutrality to oscillate at relatively high frequency of GAM (about 20-30 kHz). As for the electron temperature, there is no theoretical prediction of the electron temperature oscillations associated with the GAM development. Even if such oscillations occur, their impact on the $D_{\alpha}$ emission is expected to be very weak due to a very weak dependence of the $D_{\alpha}$ emission on the electron temperature in the range of 50 to $100 \mathrm{eV}$. Therefore it is possible to assume that the Dalpha emission oscillations at the GAM frequency are mainly dependent on the plasma density oscillations with the same frequency. The oscillations of ion saturation current of Langmuir probe at the GAM frequency were found to be in phase with the

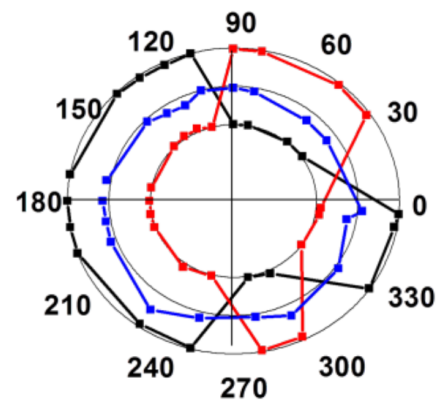

Fig. 3: Normalized magnitudes of Mirnov coil signals at GAM frequency as a function of poloidal angle. Black curve - $t_{1}=173.936 \mathrm{~ms}$, blue curve $t_{2}=173.946 \mathrm{~ms}$, red curve $t_{3}=173.956 \mathrm{~ms} . \quad t_{3}-t_{1}=$ $0.5 / f_{\text {GAM }}$. Ohmic heating discharge \#33203. $D_{\alpha}$ emission oscillations. This fact is in line with the assumption made above. The lines of sight of $D_{\alpha}$ emission detectors are shown schematically in Fig. 1. Three vertical observation chords in the poloidal cross-section at toroidal angle $\varphi=0^{\circ}$ (a, b and c in Fig. 1) and three horizontal chords at toroidal angle $\varphi=22^{\circ}$ (d, e and f) and also one horizontal line of sight in the poloidal cross-section at toroidal angle $\varphi=180^{\circ}$ were used. The $D_{\alpha}$ emission emerged mainly in the vicinity of the separatrix for the Globus-M conditions. The poloidal mode structure of plasma density oscillations with the GAM frequency was restored by the cross-correlation analysis of all above mentioned $D_{\alpha}$ signals. It was found that all signals were oscillating in phase. Therefore we have come to conclusion that the plasma density GAM perturbations have an $m / n=0 / 0$ topology that is inconsistent with the present theoretical predictions of an $m / n=1 / 0$.

In order to study the magnetic field oscillation associated with the GAM development, poloidal and toroidal Mirnov coil arrays were used. A poloidal array of 28 Mirnov coils was placed in poloidal cross-section as shown in Fig. 1. Each probe was a pick-up coil $16 \mathrm{~mm}$ high and 8.5 $\mathrm{mm}$ in diameter. The probe resistance was $9 \Omega$, and the product of the effective probe area by 
the number of coil turns was $\mathrm{sw} \approx 63 \mathrm{~cm}^{2}$. The coils were tuned to measure themagnetic field fluctuations within a frequency band of up to $100 \mathrm{kHz}$. This poloidal Mirnov coil array was used to order to determine the poloidal number of the GAM perturbation through a reduced analysis of recorded signals [9]. The frequency of the perturbation was determined from the Fourier spectrum of the probe signal (Fig. $2-B^{M P}$ ). The digital band filtration was applied to improve the signal-tonoise ratio. Since the probe signals were close to sine curves over the time interval of our study, the phase delay of the probe signal could be determined from the approximation of the recorded signal by a harmonic function. Fig. 3 shows the reconstructed poloidal structure of the GAM perturbation for three moments of time separated by a quarter of the GAM oscillation cycle. The signals were normalized by their amplitudes and, therefore, the presented values varied in a \pm 1 interval. The magnetic field GAM perturbations had an $m=2$ topology, whereat the toroidal effect seemed to lead to a drastic deformation.

\section{Bicoherent analysis at Doppler backscattering diagnostic}

It is widely believed that GAM, as a high frequency kind of zonal flows, develops due to the Reynolds stress provided by turbulent small-scale fluctuations. On the other hand, intensive localized GAM $E \times B$ velocity oscillations can suppress the plasma turbulence through sheared rotation. These two types of mutual influence of GAM and drift turbulence can be interpreted as threewave interaction which, in turn, can be extracted via bicoherent analysis. The cross-bicoherence spectrum as a measure of phase coupling between three spectral components of signals is described by the following expressions: $b^{2}\left(f_{1}, f_{2}\right)=\frac{\left|\left\langle Y_{k}^{*}\left(f_{3}\right) Y_{i}\left(f_{1}\right) Y_{j}\left(f_{2}\right)\right\rangle\right|^{2}}{\left\langle\left|Y_{k}\left(f_{3}\right)\right|^{2}\right\rangle\left\langle\left|Y_{i}\left(f_{1}\right) Y_{j}\left(f_{2}\right)\right|^{2}\right\rangle} ; \quad f_{3}=f_{1} \pm f_{2}$ where $Y_{i}\left(f_{1}\right), Y_{j}\left(f_{2}\right), Y_{k}\left(f_{3}\right)$ are spectra of different diagnostic signals. The summed cross-bicoherence $b_{1}^{2}\left(f_{2}\right)$ is used to reveal the interaction of certain harmonic $f_{2}$ with all other spectral components is as follows: $b_{1}^{2}\left(f_{2}\right)=\frac{1}{s\left(f_{2}\right)} \sum_{f_{1}} b^{2}\left(f_{1}, f_{2}\right)$ where $s\left(f_{2}\right)$ is the number of summands.

It was found in the FT-2 tokamak using the enhanced microwave backscattering technique [10] that the summed crossbicoherence has a clearly visible peak at the GAM frequency that significantly exceeded the statistical significance level $1 / M(M$ being the number of realizations for averaging) when the crossbicoherent spectrum was computed for the following combination of spectra: $Y_{i}(f)=Y_{j}(f)=Y_{B S}(f)$ being the spectrum of sine (or cosine) signal of the IQ detector, while $Y_{k}(f)=Y_{E x B}(f)$ being the spectrum of ExB velocity recovered from the Doppler frequency shift. Nevertheless, this positive result does not mean that the mutual nonlinear influence of GAM and drift turbulence has been discovered due to strong modulation of the phase of

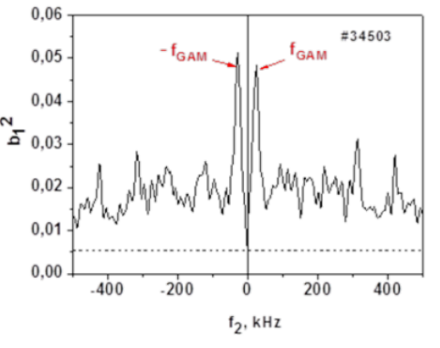

Fig. 4: Summed bicoherence $\left(b_{1}^{2}\left(f_{2}\right)\right)$. The statistical significance level is $1 / M=0.0053(M-$ number of realizations for averaging). \#34503. complex signal. To confirm this assumption, a simple simulation of the Doppler backscatter-ing was carried out [11]. The actual scattering fluctuations were substitued by a random 2D pat-tern. This turbulent media was moving with poloidal velocity $V(t)$ including the GAM veloc-ity. Subsequently this moving plasma density pattern was used to synthesize IQ detector signals: $\mathrm{I}(\mathrm{t})=\int \delta n(r, \boldsymbol{\theta}, t) W(r, \boldsymbol{\theta}) d r d \boldsymbol{\theta}$, where $W(r, \boldsymbol{\theta})$ was a complex spatial 
weighting function, $\delta n(r, \theta, t)$ was the density fluctuation and $r$ and $\theta$ were the radial and poloidal variables. Cer-tainly, there were no simulations of nonlinear processes of mutual interaction between the GAM and drift turbulence in this model. And nevertheless, the cross-bicoherent spectra computed for the same combination of synthesized signals appeared to be similar to the bispectra obtained by experimental data.

On the other hand the modeling showed that there was not any exceeding of the significant level for the spectra combination: $Y_{i}(f)=Y_{k}(f)=Y_{E x B}(f), Y_{j}(f)=Y_{A}(f)$ was the spectrum of amplitude of the complex output signal of the IQ detector The calculation of bicoherence was performed for this spectra combination by using the experimental data. The relevant summed cross-bicoherence is represented in Fig. 4.

\section{Conclusion}

In summary, we have shown that the multi-diagnostic application is very efficient for the GAM phenomena investigations. It is a combination of different methods, which makes it possible to determine the phase relationship between the GAM oscillations of various plasma parameters and restore spatial structure of GAM oscillations. It has been found that the plasma velocity, density and magnetic field oscillations at the GAM frequency have $m=0, m / n=0 / 0$ and $m=2$ topology respectively. Application of the Doppler backscattering as the main method of the GAM study has required a detailed prior consideration of the incident wave propagation in the complex geometry of spherical tokamak. A method of simple DBS simulation has been proposed, which allowed to determine the possibility of bicoherent analysis in order to reveal the mutual influence of the GAM and drift turbulence.

\section{Acknowledgments}

The study was partly supported by RFBR grant 14-02-00171.

\section{References}

[1] P.H. Diamond, et al. Plasma Phys. Control. Fusion 47 (2005) R35

[2] C. Wahlberg Plasma Phys. Control. Fusion 51 (2009) 085006

[3] Melnikov A.V., et. al., Plasma Phys. Control. Fusion 48 (2006) S87-S110

[4] G. Wang et al, Physics of Plasmas 20, (2013), 092501

[5] C.A. de Meijere, et al., Plasma Phys. Control. Fusion 56 (2014) 072001

[6] G. D. Conway et al, Plasma Phys. Control. Fusion 47 (2005) 1165-1185

[7] A.Yu. Yashin et al, Nucl. Fusion 54 (2014) 114015

[8] V. V. Bulanin et al, Plasma Physics Reports 26 (2000) 813-819

[9] M. I. Patrov et al, Plasma Physics Reports 33 (2007) 81-90

[10] A.D. Gurchenko et al, Plasma Phys. Control. Fusion 55 (2013) 085017

[11] A.Yu. Yashin et al, J. Instrum. To be published 